\title{
MicroRNA-146b-5p inhibits the growth of gallbladder carcinoma by targeting epidermal growth factor receptor
}

\author{
JINGLI CAI ${ }^{1,2}$, LUBAI XU ${ }^{2}$, ZHENZHAI CAI $^{3}$, JISHENG WANG $^{2}$, BING ZHOU $^{2}$ and HAI HU ${ }^{1}$ \\ ${ }^{1}$ Department of General Surgery, East Hospital Tongji University School of Medicine, Shanghai 200120; \\ Departments of ${ }^{2}$ General Surgery and ${ }^{3}$ Gastroenterology, The Second Affiliated Hospital of \\ Wenzhou Medical University, Wenzhou, Zhejiang 325000, P.R. China
}

Received June 7, 2014; Accepted February 20, 2015

DOI: $10.3892 / \mathrm{mmr} .2015 .3461$

\begin{abstract}
Gallbladder cancer (GBC) is the most common and aggressive type of biliary tract cancer. The study of potential treatments for $\mathrm{GBC}$ has recently focused on microRNAs, a class of small non-coding RNAs, which post-transcriptionally regulate gene expression during various crucial cell processes. The present study aimed to investigate the role of microRNA-146b (miR-146b) in GBC. Human GBC tissue and adjacent normal gallbladder tissue sections were surgically removed and miR-146b-5p expression and the development and pathological characteristics of GBC were investigated. miR-146b-5p expression was reduced in GBC tissue compared with that in adjacent tissue, and a significant correlation was observed between miR-146b-5p expression levels and carcinoma size and development. miR-146b-5p overexpression in the SGC-996 GBC cell line inhibited cell growth through enhanced apoptosis and $G_{1}$ phase arrest. Furthermore, it was demonstrated that epidermal growth factor receptor (EGFR) was regulated directly by miR-146b-5p and was essential as a mediator of the biological effects of miR-146b-5p in GBC. Enforced expression of EGFR reversed the ability of miR-146b-5p to inhibit proliferation. In conclusion, the present study indicated that the mechanism of action of miR-146b-5p in GBC involves the regulation of EGFR expression.
\end{abstract}

Correspondence to: Dr Lubai Xu, Department of General Surgery, The Second Affiliated Hospital of Wenzhou Medical University, 109 Xueyuanxi Road, Wenzhou, Zhejiang 325000, P.R. China E-mail: xulubai1966@hotmail.com

Professor Hai Hu, Department of General Surgery, East Hospital Tongji University School of Medicine, 150 Jimo Road, Shanghai 200120, P.R. China

E-mail: huhai1968@hotmail.com

Key words: microRNA-146b-5p, gallbladder cancer, epidermal growth factor receptor

\section{Introduction}

Gallbladder cancer (GBC) is the most common cancer of the biliary tract and has a particularly high incidence in humans (1). According to the United States Centers for Disease Control and Prevention, GBC is the sixth most common type of gastrointestinal cancer in the USA and >10,000 novel cases of gastric cancer are diagnosed each year. In addition, the incidence of GBC appears to increase with age and females are affected 2-6 fold more frequently than males (2). GBC is characterized by local invasion, extensive regional lymph node metastasis, vascular encasement and distant metastases. The five-year survival rates are $5-10 \%$ for patients with a GBC diagnosis, and this poor prognosis is associated with the commonly advanced stage at diagnosis due to the lack of effective screening programs and non-specific symptoms (3).

At present, complete surgical resection is the only potentially curative treatment for GBC. However, few patients are eligible for this surgery due to the advanced stage at diagnosis (4). Although there have been significant advances in the management of GBC over the previous decade, the majority of treatments are palliative and aimed at improving quality of life through relief of pain and jaundice and by potentially prolonging survival time (5). In order to improve treatment options for GBC, an improved understanding of the molecular mechanisms underlying carcinogenesis in the gall bladder is required.

MicroRNAs (miRNAs) are a class of small, single-stranded noncoding RNAs, consisting of 19-24 nucleotides, which regulate gene expression by silencing messenger RNAs (mRNAs) via binding to their 3'-untranslated region (3'UTR) (6). miRNAs are involved in a variety of biological processes, including cellular differentiation, apoptosis, metabolism and proliferation, by targeting different genes (7). Accumulating lines of evidence have indicated that miRNAs exhibit aberrant expression patterns and functional abnormalities in numerous types of cancer, including GBC. Dysregulated miRNAs are common in GBC and contribute to gallbladder carcinogenesis via the alteration of cell growth, cell cycle, apoptosis and cell migration (8-10). Human miR-146b-5p is located within 10q24-26 (104186259-104186331+), an area in which genetic material has been observed to be frequently deleted in cancer cells (11). Previous evidence has also indicated that miR-146b-5p is able 
to function as a tumor suppressor in pancreatic and breast cancer $(12,13)$. To date, despite the recent findings regarding miR-146b-5p and its important roles in carcinogenesis, no studies investigating an association between miR-146b-5p and GBC have been performed, to the best of our knowledge.

The present study aimed to examine the expression levels of miR-146b-5p in order to evaluate the clinical characteristics of miR-146b-5p expression in GBC and to evaluate the effects of aberrant miR-146b-5p expression on GBC cell lines.

\section{Patients and methods}

Patients and tissue samples. Human GBC samples $(\mathrm{n}=46)$ were obtained from a random sample of patients who had undergone curative resection at the Department of General Surgery, East Hospital Tongi University School of Medicine (Shanghai, China), between October 2012 and October 2013. All samples were immediately frozen in liquid nitrogen. None of the patients had been previously treated with radiotherapy or chemotherapy prior to the surgery. The GBC tissues from the surgical resections of the 46 patients were all verified by pathologists in the hospital. All samples were obtained with informed consent of the patients or their families, and the study protocol was approved by the Ethics Committee of the East Hospital.

RNA extraction and miRNA expression assay. For the analysis of miRNA in tissue samples using reverse transcription quantitative polymerase chain reaction (RT-qPCR) assay, total RNA was isolated using a mirVana ${ }^{\mathrm{TM}}$ miRNA isolation kit (Applied Biosystems, Foster City, CA, USA). RT and PCR amplification were performed using a TaqMan MicroRNA assay (Applied Biosystems) according to manufacturer's instructions. The primer sequences were synthesized by Applied Biosystems, and the PCR cycles comprised 40 cycles of 15 or $30 \mathrm{sec}$ at $98^{\circ} \mathrm{C}, 90 \mathrm{sec}$ at $58^{\circ} \mathrm{C}$ and $30 \mathrm{sec}$ at $72^{\circ} \mathrm{C}$; with a final extension at $72^{\circ} \mathrm{C}$ for $10 \mathrm{~min}$. All cycles were performed on an Eppendorf real time PCR machine 5331 (Eppendorf, Hamburg, Germany). All reactions, including those for the blank controls, were assessed for amplification success on a $1.5 \%$ agarose gel (Sigma-Aldrich, St. Louis, MO, USA) and visualized using a SYBR ${ }^{\circledR}$ Safe (Invitrogen Life Technologies, Carslbad, CA, USA The relative quantification of miR-146b-5p was calculated using the $2^{-\Delta \Delta \mathrm{Ct}}$ method normalized with RNU6B as the internal control and relative to a calibrator sample as the external control.

Cell culture and generation of stably transfected cell lines. The SGC-996 human GBC cell line was obtained from the American Type Culture Collection (Manassas, VA, USA) and grown in Dulbecco's modified Eagle's medium (Gibco-BRL, Grand Island, NY, USA) with $10 \%$ fetal bovine serum (Hyclone, Logan, UT, USA) in a cell culture incubator at $37^{\circ} \mathrm{C}$ and with $5 \% \mathrm{CO}_{2}$. To generate the stable miR-146b-5p-transfected cell line, the miR-146b-5p gene was amplified from human genomic DNA using Accuprime Taq polymerase (Invitrogen Life Technologies) and cloned into pMSCV-PIG vectors (Clontech Laboratories, Mountain View, CA, USA). SGC-996 cells were infected with retroviruses generated in Phoenix cells (American Type Culture
Collection), as described previously (14). After $72 \mathrm{~h}$,the cells were selected with $2 \mu \mathrm{g} / \mathrm{ml}$ puromycin (Sigma-Aldrich). The primer sequences used to amplify the miR-146b-5p gene were as follows: Forward, 5'-TGACCCATCCTGGGCCTCAA-3' and reverse, 5'-CCAGTGGGCAAGATGTGGGCC-3'.

Cell proliferation assay. The cells were plated onto 96-well microplates $\left(10^{4}\right.$ cells/well $)$ and cell viability was monitored using an MTT assay (Sigma-Aldrich), as described previously (15). The absorbance value (A) at $570 \mathrm{~nm}$ was read using a Bio-Rad 3550 (Bio-Rad Laboratories, Inc., Hercules, CA, USA). Three independent experiments were performed and mean values were calculated as the final result for comparison

Cell cycle analysis. For cell cycle analysis, flow cytometry was used. Briefly, the cells were harvested by trypsinization, washed with cold phosphate-buffered saline (PBS) and fixed with $70 \%$ cold ethanol (Sigma-Aldrich) at $4^{\circ} \mathrm{C}$ overnight. Subsequently, the fixed cells were collected, washed in PBS and stained with propidium iodide (PI; Sigma-Aldrich) in the presence of RNAse A (Sigma-Aldrich). Cell cycle analysis was performed using a FACS/Calibur flow cytometer using the CellQuest or ModFit 3.0 software packages (BD Bioscienciences, Franklin Lakes, NJ, USA).

Apoptotic assay. To evaluate apoptosis, an Annexin V-fluorescein isothiocyanate (FITC) apoptosis kit (Cell Signaling Technology, Inc., Boston, MA, USA) was used. Briefly, the cells were harvested and washed with cold PBS and resuspended in binding buffer, followed by incubation with Annexin-V/FITC and PI buffers for $15 \mathrm{~min}$ at $4^{\circ} \mathrm{C}$ in the dark. Annexin-V/FITC and PI signals were detected using flow cytometry.

Xenograft model. For xenograft experiments, 5-6-week-old male BALB/c and nude mice (on a BALB/c background), free of specific pathogens, were purchased from the Animal Laboratory of Tongji University School of Medicine. The mice were bred under accredited specific pathogen-free conditions in separate filter-top cages, and were acclimated for at least 1 week prior to treatment. The Balb/c nude mice (5-6 weeks old, male, $20 \mathrm{~g}$ ) were inoculated subcutaneously into the flank with $1 \times 10^{7}$ cells suspended in $0.1 \mathrm{ml}$ PBS. The tumor volumes were measured every week using a Vernier caliper (Fisher, Pittsburgh, PA, USA) to measure the maximal tumor diameter (L) and transverse diameter (W). The total tumor volume was calculated as: (L x W2) / 2. After 6 weeks, the mice were anesthetized with $2 \%$ sodium pentobarbital $(10 \mu \mathrm{l} / \mathrm{g}$ body weight) and then sacrificed via cervical dislocation. The tumors were removed and weighed. All procedures were performed in accordance with the Guidelines of the Chinese Association for Laboratory Animal Science.

Western blot analysis. Cells were lysed on ice in radioimmunoprecipitation assay lysis buffer (Sigma-Aldrich), containing $150 \mathrm{mM} \mathrm{NaCl}, 1 \% \mathrm{NP} 40,0.5 \%$ sodium deoxycholate, $0.1 \%$ SDS, $50 \mathrm{mM}$ Tris (pH 7.9), $10 \mathrm{mM} \mathrm{NaF}$, phenylmethylsulfonyl fluoride and $1 \mathrm{X}$ protease inhibitors. The protein concentrations were measured with a bicinchoninic acid 
Table I. Association between miR-146b-5p expression in gallbladder cancer and clinical characteristics.

\begin{tabular}{|c|c|c|c|c|}
\hline \multirow[b]{2}{*}{ Characteristic } & \multirow[b]{2}{*}{ Cases (n) } & \multicolumn{2}{|c|}{ Relative miR-146b-5p expression } & \multirow[b]{2}{*}{ P-value } \\
\hline & & Low $(\mathrm{n})$ & $\operatorname{High}(\mathrm{n})$ & \\
\hline Gender & & & & 0.7672 \\
\hline Male & 23 & 12 & 11 & \\
\hline Female & 23 & 13 & 10 & \\
\hline Age (years) & & & & 0.3447 \\
\hline$>60$ & 25 & 16 & 9 & \\
\hline$\leqq 60$ & 21 & 10 & 11 & \\
\hline Size of carcinoma $(\mathrm{cm})$ & & & & 0.0083 \\
\hline$>3$ & 26 & 18 & 8 & \\
\hline$\leqq 3$ & 20 & 6 & 14 & \\
\hline Degree of differentiation & & & & 0.0191 \\
\hline Well and moderately differentiated & 21 & 7 & 14 & \\
\hline Poorly differentiated & 25 & 17 & 8 & \\
\hline TNM stage & & & & 0.3514 \\
\hline I-II & 20 & 8 & 12 & \\
\hline III-IV & 26 & 14 & 12 & \\
\hline Lymph node metastasis & & & & 0.2515 \\
\hline Negative & 27 & 11 & 16 & \\
\hline Positive & 19 & 11 & 8 & \\
\hline Distant metastasis & & & & 0.5949 \\
\hline Negative & 22 & 9 & 13 & \\
\hline Positive & 24 & 8 & 16 & \\
\hline
\end{tabular}

Based on the American joint committee on cancer/international union against cancer staging manual (2009). Differences between variables were assessed using a $\chi^{2}$ test. miR, microRNA; TNM, tumor-node-metastasis.

protein assay reagent kit (Pierce, Rockford, IL, USA). The protein extracts $(30 \mu \mathrm{g})$ were separated by $8 \%$ SDS-PAGE (Beyotime Institute of Biotechnology, Haimen, China) and then transferred onto polyvinylidene fluoride membranes. Human monoclonal epidermal growth factor receptor (EGFR) was used as the primary antibody $(1: 1,000$; Cell Signaling Technology; Cat. no. \#2646) at $4^{\circ} \mathrm{C}$ overnight. A monoclonal $\beta$-actin antibody (1:1,000; Cell Signaling Technology, Inc.; cat. no. \#8457), at $4^{\circ} \mathrm{C}$ overnight, was used as a control. The bands were detected with an enhanced chemiluminiscence kit (GE Healthcare, Little Chalfont, UK) and visualized with the ChemiDoc XRS system (Bio-Rad Laboaratories, Inc.).

Plasmid construction. To generate reporter constructs for luciferase assays, the full-length 3'UTR of EGFR, as well as the mutant (Mut) sequence of EGFR, were synthesized using PCR. The used primers contained the following restriction sites: EGFR, 3'UTR forward, 5'-GGGG TACCCCACGGAGGATAGTATGAGCCC-3' and reverse, 5'-GAAGATCTTCAGAGTGGAAATGAATATAGTT-3'; and Mut Rab23 3'UTR forward, 5'-GTTTGTGTTACTTCTAAA AGATAGTTTTCT-3' and reverse, 5'-AGAAAACTATCTTTT AGAAGTAACACAAAC-3'.

The PCR product of the EGFR 3'UTR was cloned into the KpnI and $B g l \mathrm{II}$ restriction sites downstream of the open reading frame of luciferase in a pGL3-promoter luciferase vector (Invitrogen Life Technologies).

The 3'UTR deletion of EGFR was amplified via PCR with the following primer sequences: EGFR forward, 5'-GCAGCGATGCGACCCTCCGGGACGGCC-3' and reverse, 5'-CAGTGAATTTATTGGAGCATGACCAC-3'. The resulting PCR amplicons of EGFR were cloned into the $\mathrm{T}$ vector (Promega, Madison, WI, USA). The correct clones were confirmed by sequencing.

Luciferase assays. SGC-996 cells were seeded onto a 24-well plate $\left(1.5 \times 10^{5}\right.$ per well $)$ and co-transfected with miRNA mimics (50 nM; Dharmacon, Lafayette, CO, USA) and plasmid (200 ng) using Lipofectamine ${ }^{\mathrm{TM}} 2000$ reagent (Invitrogen Life Technologies) according to the manufacturer's instructions. The luciferase activity was analyzed using dual luciferase assays (Promega) after $48 \mathrm{~h}$ of co-transfection and normalized against Renilla luciferase gene activity.

Statistical analysis. SPSS 17.0 software (SPSS, Inc., Chicago, IL, USA) was used for statistical analysis. Values are expressed as the mean \pm standard deviation of at least three repeated individual experiments for each group. Significant differences were determined using Student's t-test or $\chi^{2}$ analysis for comparisons between two groups and one-way analysis of 
A

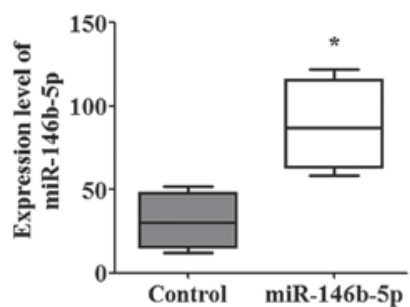

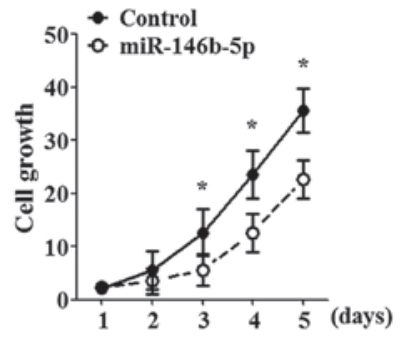

Figure 1. miR-146b-5p inhibits the growth of SGC-996 cells. (A) Mature miR-146b-5p expression was measured by reverse transcription quantitative polymerase chain reaction. Values are presented as a box-whisker plot. The horizontal line in each box indicates the median value of miR-146b-5p normalized to RNU6B. Boxes indicate the 50th and 75th percentile range of scores and the whiskers indicate the highest and lowest values. Values are presented as the mean \pm standard deviation $(n=4)$. ${ }^{*} \mathrm{P}<0.05$ versus control. (B) Cell viability of SGC-996 cells was measured using an MTT assay. Cell viability was calculated using the formula: $\left[\left(A_{E}-A_{B}\right) /\left(A_{C}-A_{B}\right)\right]$, with $A_{E}, A_{C}$ and $A_{B}$ defined as the absorbance of the experimental samples, untreated samples and blank controls, respectively. Values are expressed as the mean \pm standard deviation $(n=3)$. $P<0.05$ versus control. miR, microRNA.

variance or the non-parametric Kruskal-Wallis H test was used for multiple comparisons. $\mathrm{P}<0.05$ was considered to indicate a statistically significant difference.

\section{Results}

Elevated miR-146b-5p expression inhibits GBC growth. To investigate the pathogenicity of miR-146b-5p in the development and progression of GBC, 46 GBC tissue samples were analyzed using TaqMan RT-qPCR. The clinicopathological data for 46 patients are shown in Table I. Based on the overall expression levels of miR-146b-5p, the GBC specimens were divided into two groups (a high miR-146b-5p expression group and a low expression group). Elevated miR-146b-5p expression was observed in patients with a smaller tumor size and well-differentiated tumors. No significant difference was observed when comparing the groups with any other clinicopathological feature, including gender, age, tumor-node-metastasis stage and metastasis. Subsequently, the anti-tumorigenic function of miR-146b-5p in human GBC cell lines was examined by generating stable cell lines expressing miR-146b-5p. SGC-996 cells overexpressing miR-146b-5p exhibited a 2.5 -fold increase in miR-146b-5p expression compared with that in the control cells (Fig. 1A). Functional studies revealed that miR-146b-5p overexpression significantly inhibited the growth of SGC-996 cells and this effect increased with time (Fig. 1B).

miR-146b-5p induces $G_{1}$ arrest and apoptosis in $S G C-996$ cells. To further elucidate the growth inhibition mechanism of miR-146b-5p in SGC-996 cells, the cell cycle stage and apoptotic levels were analyzed. The overexpression of miR-146b-5p in SGC-996 cells significantly increased the percentage of cells in $G_{0} / G_{1}$ phase compared with that in the control group (Fig. 2A). In addition, compared with the that in the control group, the apoptotic rate of SGC-996 cells in miR-146b-5p-overexpressing cells was markedly increased (Fig. 2B).

miR-146b-5p inhibits tumor growth in vivo. To confirm the effect of miR-146b-5p in vivo, a GBC-bearing nude mouse model was generated. miR-146b-5p-overexpressing and control cells were injected subcutaneously into the flanks of nude mice. miR-146b-5p suppressed the growth of the tumor, as indicated by decreased tumor weight and volume as compared with that in the controls. Differences between the miR-146b and control group were significant and increased with time (Fig. 3A-C).

EGFR is a direct target of miR-146b-5p in SGC-996 cells. As miRNA functions primarily by inhibiting target genes, the target of miR-146b-5p in GBC was examined. miR-146b-5p has recently been proposed to suppress EGFR expression by binding to the 3'UTR of EGFR in glioma (16). To investigate whether miR-146b-5p decreased EGFR expression in GBC, SGC-996 cells were transfected with miR-146b-5p mimics. Western blot analysis also confirmed that transfection with miR-146b-5p mimics significantly suppressed the expression of EGFR protein in SGC-996 cells (Fig. 4A). To confirm that the inverse correlation between miR-146b-5p and EGFR expression in SGC-996 cells was due to a direct interaction, the potential seed sequence for miR-146b-5p was analyzed in the 3'UTR region of EGFR, and the wild-type and mutant EGFR 3'UTR fragments were cloned into a luciferase reporter gene system (Fig. 4B). miR-146b-5p mimics and luciferase reporter plasmids were co-transfected into SGC-996 cells. Of note, it was identified that the activity of a luciferase reporter gene linked to the wild-type EGFR 3'UTR fragment decreased in response to transfection with miR-146b-5p mimics (Fig. 4C). By contrast, miR-146b-5p had no effect on the activity of the luciferase reporter gene cloned into the mutant EGFR 3'UTR region (Fig. 4C).

Ectopic expression of EGFR is able to reverse the growth inhibition caused by miR-146b-5p. EGFR was recently hypothesized to have a crucial role in GBC growth (17). To demonstrate whether miR-146b-5p functions were mediated through EGFR, expression vectors for EGFR lacking the respective 3'UTR or empty vector were transfected into miR-146b-5p-overexpressing cells. Western blot analysis demonstrated an increased expression of EGFR proteins in SGC-996 cells transfected with miR-146b-5p and EGFR compared with those transfected with the empty vector (Fig. 5A). Cells overexpressing miR-146b-5p exhibited an apparent rescue of SGC-996 cell proliferation, as revealed using an MTT assay, following recovery of EGFR expres- 
A
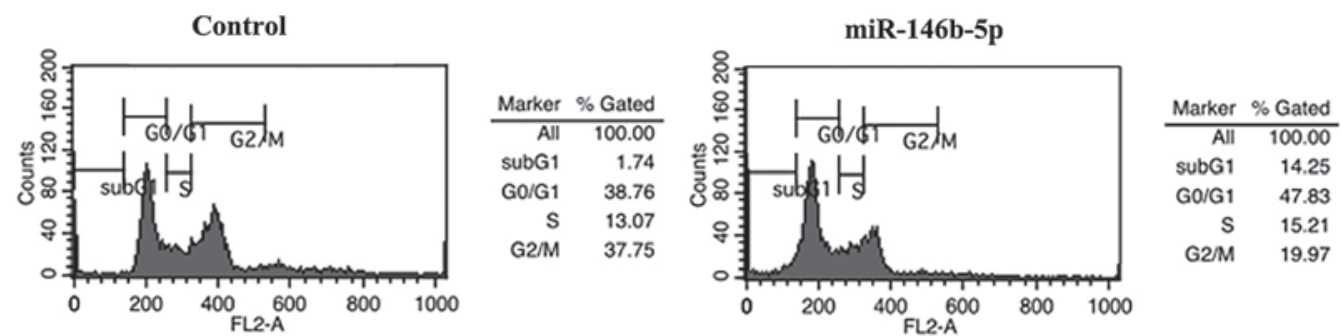

B
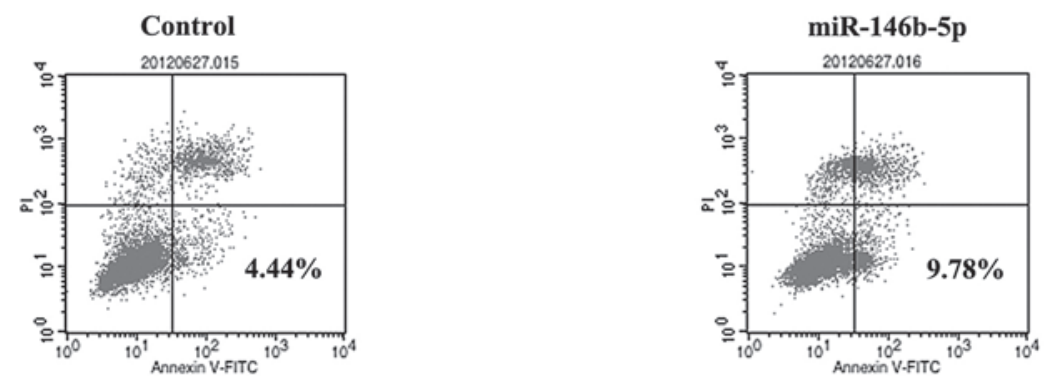

Figure 2. miR-146b-5p induces $\mathrm{G}_{1}$ arrest and apoptosis in SGC-996 cells. (A) Cell cycle and (B) apoptosis were analyzed by flow cytometry in control and miR-146b-5p-overexpressing SGC-996 cells. Diagrams are representative of three independent experiments. miR, microRNA; FITC, fluorescein isothiocyanate.

A

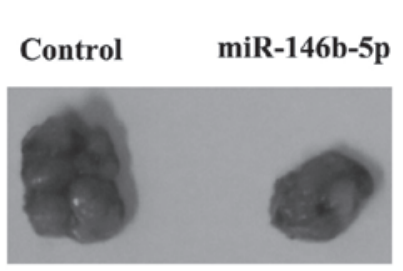

B

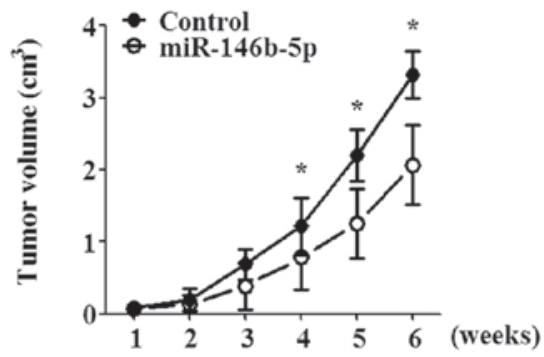

C

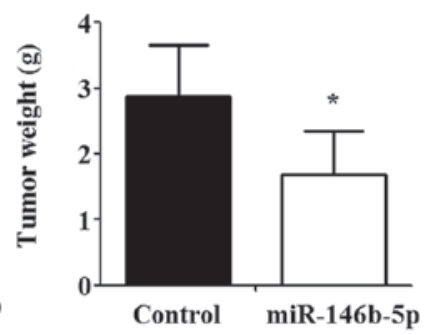

Figure 3. Overexpression of miR-146b-5p suppresses tumor growth in vivo. Nude mice received subcutaneous injections of the indicated cells. The mice were sacrificed at the end of the experiment (six weeks). (A) Macroscopic xenograft tumors in nude mice generated by subcutaneous injection of SGC-996 cells. (B) Growth curves of xenografts. Tumor volume was measured each week. Values are expressed as the mean \pm standard deviation ( $\mathrm{n}=3$ ). ${ }^{*} \mathrm{P}<0.05$ versus control,. (C) The tumor weight was measured following sacrification of the mice. Values are expressed as the mean \pm standard deviation ( $\mathrm{n}=3$ ). ${ }^{*} \mathrm{P}<0.05$ versus control. miR, microRNA.

A

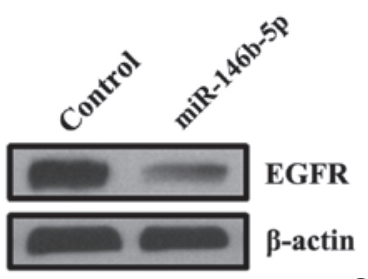

B

\begin{abstract}
EGFR 3'UTR
miR-146b-5p
\end{abstract}

Mut EGFR 3'UTR

miR-146b-5p

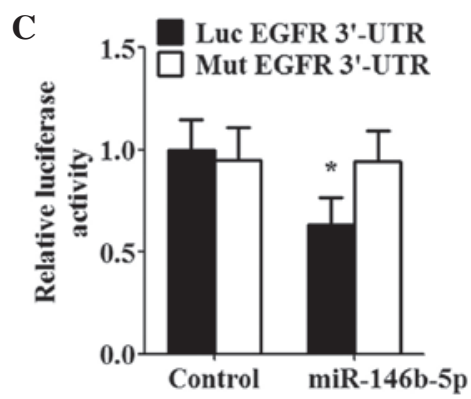

5'......GTGTTACTTATGGAAGATAGT......3'

3'......UCGGAUUÁCCUUUAAGUCAAGAGU......5"

5'.....GTGTTACTTCTAAAAGATAGT.......3'

3'......UCGGAUUACCUUAAGUCAAGAGU......5"

Figure 4. miR-146b-5p reduces EGFR expression in SGC-996 cells. (A) Western blot analysis of EGFR and $\beta$-actin expression in the indicated cell extracts (B) Full-length 3'UTR sequences of wild type EGFR and the mutant sequence of EGFR were cloned into pGL3-promoter luciferase vector. The predicted base-pairing between miR-146b-5p and the 3'UTR sequences of EGFR are shown (solid lines indicate binding sites and cross indicate low-affinity matches). (C) SGC-996 cells were co-transfected with reporter plasmid and miR-146b-5p mimics. Values are presented as the mean \pm standard deviation. ${ }^{*} \mathrm{P}<0.05$ versus wild type group $(n=3)$. EGFR, epidermal growth factor receptor; UTR, untranslated region; miR, microRNA; mut, mutant. 


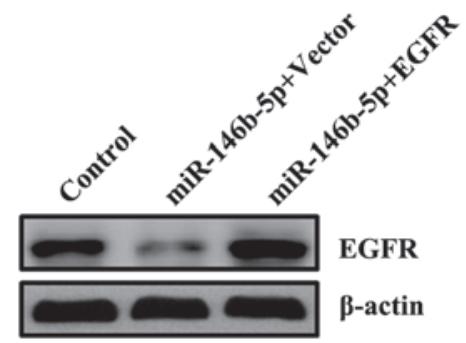

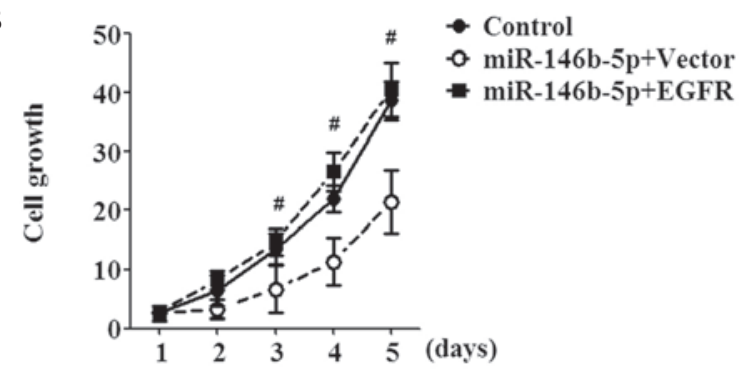

C

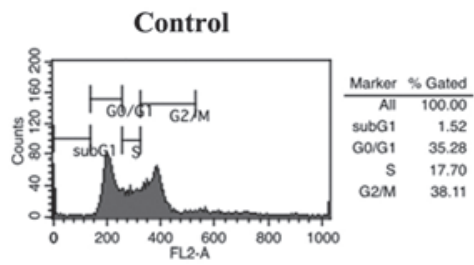

D

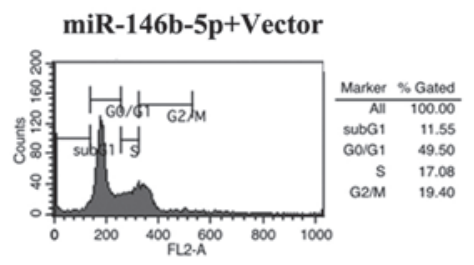

miR-146b-5p+Vector

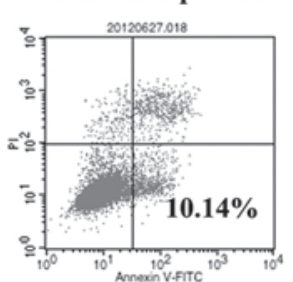

miR-146b-5p+EGFR

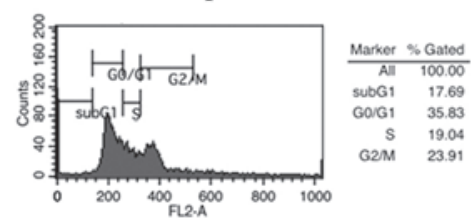

miR-146b-5p+EGFR

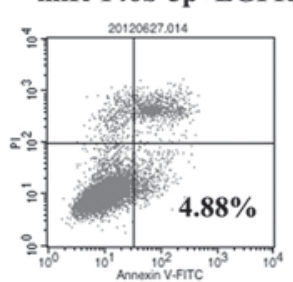

Figure 5. Growth inhibitory properties of miR-146b-5p may be reversed by co-expression of EGFR. Cells were transfected with empty vector and EGFR expression plasmid for $48 \mathrm{~h}$. (A) Western blot analysis of EGFR $48 \mathrm{~h}$ after transfection with $\beta$-actin as the loading control. (B) Cell viability was determined using an MTT assay. Values are presented as the mean \pm standard deviation. ${ }^{~} \mathrm{P}<0.05$, versus miR-146b-5p + vector group (n=3). (C) Cell cycle and (D) apoptosis were analyzed by flow cytometry $48 \mathrm{~h}$ after transfection. Data are representative of three independent experiments. miR, microRNA; FITC, fluorescein isothiocyanate; EGFR, epidermal growth factor receptor.

sion and no significant differences were observed compared with the control (Fig. 5B). The cell cycle and apoptosis assays revealed that EGFR overexpression decreased the percentage of cells in $\mathrm{G}_{0} / \mathrm{G}_{1}$ phase, decreased the rate of apoptosis and reversed the growth inhibitory effect of miR-146b-5p (Fig. 5C and D).

\section{Discussion}

As one of the most frequent malignant neoplasms of the biliary tract system, GBC is characterized by late clinical presentation and diagnosis, with limited treatment options and poor prognosis (4). Although the incidence of GBC has appeared to increase, the molecular mechanisms that cause GBC carcinogenesis remain to be elucidated, and the available treatment methods have not significantly improved the survival rates of affected patients.

In the present study, it was identified that the expression of miR-146b-5p was associated with tumor size and differentiation stage in patients, and that tumors of patients with lower levels of miR-146b-5p tended to be larger and poorly differentiated. However, no difference in miR-146b-5p expression was observed regarding the presence or absence of metastasis, indicating that miR-146b-5p is not involved in the regulation of GBC metastasis. These results indicated that miR-146b-5p expression may be an important indicator for GBC carcinogenesis and growth.
EGFR belongs to the ErbB family of receptor tyrosine kinases, which are activated by several ligands, including EGF, transforming growth factor- $\alpha$, amphiregulin, heparin-binding EGF, betacellulin and epiregulin (18). The EGFR signaling pathway exerts its biological effects via multiple signaling cascades and is crucial in tumor growth (19-21). Previous studies have demonstrated that EGFR overexpression is common in GBC and that inhibiting EGFR signaling results in considerable anti-proliferative effects on in vitro models of $\operatorname{GBC}(22,23)$. In the present study, a significant inverse correlation was observed between miR-146b-5p expression levels and EGFR protein in human GBC cell lines. In addition, it was demonstrated that EGFR was negatively regulated by miR-146b-5p via binding to the 3'UTR of EGFR mRNA in vitro and the inhibitory effect of miR-146b-5p on proliferation may be reversed by overexpression of EGFR. The present study demonstrated that miR-146b-5p inhibited GBC growth, with concomitant suppression of EGFR.

Despite numerous studies aiming to elucidate the mechanisms involved in GBC, the treatment of GBC remains a major challenge in oncology. Molecularly targeted agents, which inhibit EGFR pathways are being assessed in clinical trials and appear to be a potent therapeutic target for patients with GBC. The findings of the present study, regarding miR-146b-5p and EGFR in GBC are in accordance with those of previous studies, which demonstrated that miR-146b-5p decreased EGFR expression in the MDA-MB-231 breast cancer cell line 
and the U87-MG glioma cell line $(12,16,24)$, elucidating the function of miR-146b-5p in GBC. It was demonstrated in the present study that miR-146b-5p is an essential miRNA, which regulates GBC growth and relies on the EGFR signaling pathway. The manipulation of miR-146b-5p may be an effective therapeutic target for GBC patients in the future.

\section{Acknowledgements}

The present study was supported by the Zhejiang Provincial Natural Science Foundation of China (grant no. Y2110335).

\section{References}

1. Zhu AX, Hong TS, Hezel AF and Kooby DA: Current management of gallbladder carcinoma. Oncologist 15: 168-181, 2010.

2. Hueman MT, Vollmer CM Jr and Pawlik TM: Evolving treatment strategies for gallbladder cancer. Ann Surg Oncol 16 : 2101-2115, 2009

3. de Groen PC, Gores GJ, LaRusso NF, Gunderson LL and Nagorney DM: Biliary tract cancers. N Engl J Med 341: $1368-1378,1999$

4. Zhang LQ, Zhang XD, Xu J, et al: Potential therapeutic targets for the primary gallbladder carcinoma: estrogen receptors. Asian Pac J Cancer Prev 14: 2185-2190, 2013.

5. McNamara MG, Metran-Nascente C and Knox JJ: State-of-the-art in the management of locally advanced and metastatic gallbladder cancer. Curr Opin Oncol 25: 425-431, 2013.

6. Farh KK, Grimson A, Jan C, et al: The widespread impact of mammalian MicroRNAs on mRNA repression and evolution Science 310: 1817-1821, 2005.

7. Wu WK, Lee CW, Cho CH, et al: MicroRNA dysregulation in gastric cancer: a new player enters the game. Oncogene 29: 5761-5771, 2010

8. Peng HH, Zhang YD, Gong LS, Liu WD and Zhang Y: Increased expression of microRNA-335 predicts a favorable prognosis in primary gallbladder carcinoma. Onco Targets Ther 6: $1625-1630,2013$

9. Kono H, Nakamura M, Ohtsuka T, et al: High expression of microRNA-155 is associated with the aggressive malignant behavior of gallbladder carcinoma. Oncol Rep 30: 17-24, 2013.

10. Chang Y, Liu C, Yang J, et al: MiR-20a triggers metastasis of gallbladder carcinoma. J Hepatol 59: 518-527, 2013.
11. Rasheed BK, Fuller GN, Friedman AH, Bigner DD and Bigner SH: Loss of heterozygosity for 10q loci in human gliomas. Genes Chromosomes Cancer 5: 75-82, 1992.

12. Hurst DR, Edmonds MD, Scott GK, Benz CC, Vaidya KS and Welch DR: Breast cancer metastasis suppressor 1 up-regulates miR-146, which suppresses breast cancer metastasis. Cancer Res 69: 1279-1283, 2009.

13. Lin F, Wang X, Jie Z, et al: Inhibitory effects of miR-146b-5p on cell migration and invasion of pancreatic cancer by targeting MMP16. J Huazhong Univ Sci Technolog Med Sci 31: 509-514, 2011.

14. Datta J, Majumder S, Kutay H, et al: Metallothionein expression is suppressed in primary human hepatocellular carcinomas and is mediated through inactivation of CCAAT/enhancer binding protein alpha by phosphatidylinositol 3-kinase signaling cascade. Cancer Res 67: 2736-2746, 2007.

15. Nasser MW, Datta J, Nuovo G, et al: Down-regulation of micro-RNA-1 (miR-1) in lung cancer. Suppression of tumorigenic property of lung cancer cells and their sensitization to doxorubicin-induced apoptosis by miR-1. J Biol Chem 283: 33394-33405, 2008.

16. Katakowski M, Zheng X, Jiang F, Rogers T, Szalad A and Chopp M: MiR-146b-5p suppresses EGFR expression and reduces in vitro migration and invasion of glioma. Cancer Invest 28: 1024-1030, 2010.

17. Harder J, Waiz O, Otto F, et al: EGFR and HER2 expression in advanced biliary tract cancer. World J Gastroenterol 15: 4511-4517, 2009.

18. Harris RC, Chung E and Coffey RJ: EGF receptor ligands. Exp Cell Res 284: 2-13, 2003.

19. Ménard S, Casalini P, Campiglio M, Pupa SM and Tagliabue E: Role of HER2/neu in tumor progression and therapy. Cell Mol Life Sci 61: 2965-2978, 2004.

20. Aaronson SA: Growth factors and cancer. Science 254: $1146-1153,1991$

21. Hudis CA: Trastuzumab-mechanism of action and use in clinical practice. N Engl J Med 357: 39-51, 2007.

22. Harder J, Waiz O, Otto F, et al: EGFR and HER2 expression in advanced biliary tract cancer. World J Gastroenterol 15: 4511-4517, 2009.

23. Pignochino Y, Sarotto I, Peraldo-Neia C, et al: Targeting EGFR/HER2 pathways enhances the antiproliferative effect of gemcitabine in biliary tract and gallbladder carcinomas. BMC Cancer 10: 631, 2010.

24. Bhaumik D, Scott GK, Schokrpur S, Patil CK, Campisi J and Benz CC: Expression of microRNA-146 suppresses NF-kappaB activity with reduction of metastatic potential in breast cancer cells. Oncogene 27: 5643-5647, 2008. 